\title{
ERS/EAACI statement on adherence to international adult asthma guidelines
}

\author{
Alexander G. Mathioudakis $\mathbb{1}^{1,2,21}$, Olympia Tsilochristou ${ }^{3,4,21}$, Ian M Adcock $\mathbb{D}^{5}$, Andras Bikov ${ }^{1,2}$, \\ Leif Bjermer ${ }^{6}$, Enrico Clini $\mathbb{1}^{7}$, Breda Flood ${ }^{8}$, Felix Herth $\mathbb{1}^{9}$, Ildiko Horvath ${ }^{10,11}$, Omer Kalayci ${ }^{12}$, \\ Nikolaos G. Papadopoulos ${ }^{1,13}$, Dermot Ryan ${ }^{10}{ }^{14}$, Silvia Sanchez Garciaa ${ }^{15}$, Jaime Correia-de-Sousa ${ }^{16,17}$, \\ Thomy Tonia ${ }^{18}$, Hillary Pinnock ${ }^{14}$, loana Agache ${ }^{19,21}$ and Christer Janson (10) 20,21
}

\begin{abstract}
${ }^{1}$ Division of Infection, Immunity and Respiratory Medicine, School of Biological Sciences, University of Manchester, Manchester, UK. ${ }^{2}$ North West Lung Centre, Wythenshawe Hospital, Manchester University NHS Foundation Trust, Manchester, UK. ${ }^{3}$ Dept of Allergy, Guy's and St Thomas' Foundation Trust, London, UK. ${ }^{4}$ Peter Gorer Dept of Immunobiology, King's College London, London, UK. ${ }^{5}$ National Heart and Lung Institute, Imperial College London and the NIHR Imperial Biomedical Research Centre, London, UK. ${ }^{6}$ Respiratory Medicine and Allergology, Dept of Clinical Sciences, Lund University, Lund, Sweden. ${ }^{7}$ Dept of Medical Specialities, University Hospital of Modena, University of Modena-Reggio Emilia, Modena, Italy. ${ }^{8}$ European Federation of Allergy and Airways Diseases Patients Association (EFA), Dublin, Ireland. ${ }^{9}$ Dept of Pneumology and Critical Care Medicine, Thoraxklinik and Translational Lung Research Center Heidelberg, University of Heidelberg, Heidelberg, Germany. ${ }^{10}$ National Koranyi Institute for Pulmonology, Budapest, Hungary. ${ }^{11}$ Institute of Public Health, Semmelweis University, Budapest, Hungary. ${ }^{12}$ Hacettepe University School of Medicine, Ankara, Turkey. ${ }^{13}$ Allergy Dept, Paediatric Clinic, National and Kapodistrian University of Athens, Athens, Greece. ${ }^{14}$ Asthma UK Centre for Applied Research, Usher Institute, University of Edinburgh, Edinburgh, UK. ${ }^{15}$ Allergy Dept, University Children's Hospital Niño Jesus, Madrid, Spain. ${ }^{16}$ Life and Health Sciences Research Institute (ICVS), School of Medicine, University of Minho, Braga, Portugal. ${ }^{17}$ ICVS/3B's - PT Government Associate Laboratory, Guimarães, Portugal. ${ }^{18}$ Institute of Social and Preventive Medicine, University of Bern, Bern, Switzerland. ${ }^{19}$ Allergy \& Clinical Immunology, Transylvania University, Brasov, Romania. ${ }^{20}$ Dept of Medical Science, Respiratory, Allergy and Sleep Research, Uppsala University and University Hospital, Uppsala, Sweden. ${ }^{21}$ These authors were Task Force Co-chairs and are equal authors.
\end{abstract}

Corresponding author: Alexander Mathioudakis (a.mathioudakis@nhs.net)

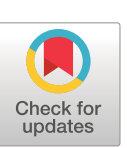

This version is distributed under the terms of the Creative Commons Attribution NonCommercial Licence 4.0. For commercial reproduction rights and permissions contact permissions@ersnet.org

This article has supplementary material available from err.ersjournals.com

Received: 5 June 2021 Accepted: 26 June 2021

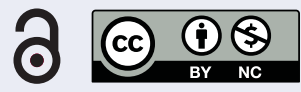

Shareable abstract (@ERSpublications)

@EuroRespSoc @AllergyEAACI statement: Guidelines need to account for differences in resource availability across various asthma care settings. Continuous, multifaceted quality improvement processes are needed to optimise and maintain guidelines adherence. https://bit.ly/2UZK5rJ

Cite this article as: Mathioudakis AG, Tsilochristou O, Adcock IM, et al. ERS/EAACl statement on adherence to international adult asthma guidelines. Eur Respir Rev 2021; 30: 210132 [DOI: 10.1183/ 16000617.0132-2021].

\section{Abstract}

Guidelines aim to standardise and optimise asthma diagnosis and management. Nevertheless, adherence to guidelines is suboptimal and may vary across different healthcare professional (HCP) groups.

Further to these concerns, this European Respiratory Society (ERS)/European Academy of Allergy and Clinical Immunology (EAACI) statement aims to: 1) evaluate the understanding of and adherence to international asthma guidelines by HCPs of different specialties via an international online survey; and 2) assess strategies focused at improving implementation of guideline-recommended interventions, and compare process and clinical outcomes in patients managed by HCPs of different specialties via systematic reviews.

The online survey identified discrepancies between HCPs of different specialties which may be due to poor dissemination or lack of knowledge of the guidelines but also a reflection of the adaptations made in different clinical settings, based on available resources. The systematic reviews demonstrated that multifaceted quality improvement initiatives addressing multiple challenges to guidelines adherence are most effective in improving guidelines adherence. Differences in outcomes between patients managed by generalists or specialists should be further evaluated.

Guidelines need to consider the heterogeneity of real-life settings for asthma management and tailor their recommendations accordingly. Continuous, multifaceted quality improvement processes are required to optimise and maintain guidelines adherence. Validated referral pathways for uncontrolled asthma or uncertain diagnosis are needed. 
Introduction

In the European Union, over 20 million people suffer from asthma [1]. During the 1990s there was a rapid decrease in asthma mortality [2], probably related to the increased use of inhaled corticosteroids (ICS) [3]. However, during the last decade, asthma mortality rates have plateaued, and a consistently high proportion of patients have uncontrolled asthma [4,5]. As a result, many patients with asthma still have impaired quality of life and suffer from chronic respiratory symptoms, often including night-time symptoms, causing sleep disturbance, excessive daytime sleepiness and decreased work productivity [6, 7].

The reason for this lack of improvement in achieving asthma control is multifactorial. Asthma is a chronic inflammatory airway disease needing regular long-term anti-inflammatory treatment for symptom control and prevention of acute attacks and/or lung function decline. ICS are the mainstay of asthma medication, but many patients do not adhere to regular treatment [8] with overreliance on short-acting $\beta$-agonists, leading to under treatment of the chronic inflammation [9]. Another possible explanation is the heterogeneity of asthma, so that subgroups of patients require different interventions, according to a personalised approach based on asthma phenotypes [10]. A proportion have severe asthma [11] and need to be identified and offered specific regimes such as biological treatment with anti-immunoglobulin (Ig)E, anti-interleukin (IL)-5 or anti-IL-4/IL-13 [12, 13]. Other factors such as poor inhaler adherence and technique, lack of self-management support, exposure to triggers, unavoidable environmental factors, limited accessibility to diagnostic facilities and medication, could also contribute [14-16].

Clinical practice guidelines, based on available evidence, define disease control and risk of acute attacks and make recommendations to standardise and optimise asthma diagnosis and management. National and international asthma guidelines have been available since the 1990s and are continuously being updated $[11,17,18]$. However, there are concerns that adherence to guidelines is far from optimal and varies between different groups of healthcare professionals (HCPs) [19, 20]. In addition, the "one-size-fits-all" approach of guidelines (typically based on efficacy in highly selected populations evaluated in randomised controlled trials (RCTs)) limits perceived applicability and relevance in real-life practice [21]. Further to these concerns, we aimed to: 1) evaluate and compare the understanding of and adherence to international asthma guidelines by HCPs of different specialties; 2) assess effectiveness of strategies aimed at improving implementation of guideline-recommended interventions; and 3) compare process and clinical outcomes in patients managed by specialists (respiratory physicians or allergists) or generalists (internists or general practitioners).

\section{Methods}

This task force was formed by the European Respiratory Society (ERS) and the European Academy of Allergy and Clinical Immunology (EAACI) in 2015 and was chaired by two representatives from the ERS (A.G. Mathioudakis and C. Janson) and two from EAACI (O. Tsilochristou and I. Agache) who were responsible for project management and co-ordination. The task force was composed of experts from three ERS Assemblies: Assembly 1 Respiratory clinical care and physiology; Assembly 5 Airway diseases, asthma, COPD and chronic cough; and Assembly 6 Epidemiology and environment. As well as from four EAACI bodies (Asthma Section, Primary Care Interest Group, Executive Committee and Junior Members Assembly) and the International Primary Care Respiratory Group (IPCRG; J. Correia-de-Sousa). It involved experts in respiratory medicine and science, allergy and general practice, and also a lay person living with the experience of asthma (B. Flood). The co-chairs met in January 2017 and September 2018 and a face-to-face meeting of the task force was held in January 2019, with teleconferences and e-mail correspondence as required. All task force members signed conflict of interest statements at the beginning of the project and updated them at project finalisation or when any new relevant conflicts appeared, in line with the ERS and EAACI procedures. This report was informed by an international online survey (Aim 1) and two systematic reviews (Aims 2 and 3).

\section{On-line survey: Aim 1}

Three online questionnaires pertaining to different clinical cases were prepared by the panel and uploaded to the SurveyMonkey platform (available in the online supplement). The cases were not related to a specific clinical setting so that the questionnaires were applicable to all specialties targeted by the survey. The first scenario was mild type 2 (T2) asthma, the second was severe T2 asthma, and the third was severe non-T2 asthma. T2 asthma is defined by the presence of eosinophilic inflammation driven via three pathways: IgE, IL-5 or IL-4/IL-13 [21]. Allergic asthma is a sub-endotype of T2 asthma, frequently with childhood onset and associated with other atopic diseases (allergic rhinitis, atopic dermatitis, food allergy). Another sub-endotype is non-allergic eosinophilic asthma, with adult-onset, which is usually more difficult to control [21]. Non-T2 asthma is usually defined by the lack of eosinophilic inflammation [21]. Its mechanisms are less well described as opposed to T2 asthma [22]. 
Introductory questions collected the participants' age, sex, specialty, level of training (trained or in training), and clinical setting. The T2 asthma questionnaires were sent out in May 2018 as a pair (mild T2 questions were completed prior to the severe T2 questions), and the non-T2 questionnaire was distributed in August 2018. Surveys were open for approximately 6 weeks. For most of the questions more than one answer could be chosen. Participants of the second survey were not asked if they had also taken part in the first survey. After completion, a participant could not take the survey again on the same computer.

Both survey links were disseminated via mass emails with links to the online surveys to relevant members of the participating organisations (EAACI: Asthma Section, ENT Section, Immunotherapy, Occupational Allergy, Allied Health and Primary Care Interest Groups, EAACI National Societies platform; ERS aforementioned assemblies; IPCRG). EAACI and ERS social media platforms supplemented the dissemination of the survey links.

Survey results were analysed based on the participants' specialty. Specialties were grouped into three main categories: 1) "allergy doctor" if the participant indicated they were an allergy-asthma specialist, allergy specialist or allergy trainee; 2) "respiratory doctor" if ther participant indicated they were an asthma specialist, respiratory doctor or respiratory medicine trainee; and 3) "generalist" if the participant indicated they were a General Practitioner, General Practitioner trainee, internist, internal medicine trainee, specialist nurse or nurse trainee.

The results of the questionnaire answers are presented as percentage of affirmative answers. Comparisons between the three groups were made using the Chi-squared test. Stata 15 (Stata Corp, College Station, TX, USA) was used for the calculations.

Ethics approval was not necessary for this survey as no personally identifiable data were collected.

\section{Systematic review methods: Aims 2 and 3}

Two systematic reviews were conducted to evaluate the: 1) effectiveness of strategies to improve adherence to guidelines on the diagnosis, assessment and long-term/acute treatment of asthma, including maintenance and acute attacks management (Aim 2); and 2) process and clinical outcomes in patients managed by specialists (respiratory physicians or allergists) compared to generalists (internists or general practitioners) (Aim 3) (table 1). The systematic reviews followed Cochrane methodology [23]. Medline/PubMed was searched for studies published after 1990 (publication of the first asthma guideline [24]), using a search strategy that included controlled vocabulary and free search terms (available in the online supplement), to identify relevant studies. Reference lists of included studies and of any previous, relevant systematic reviews were screened. Studies of any design addressing the two review questions were eligible if they assessed process outcomes (e.g. adherence to guideline recommendations) and/or asthma-related clinical outcomes. Two reviewers independently evaluated all identified abstracts for eligibility. The full texts of all potentially eligible manuscripts were similarly evaluated for inclusion by two reviewers. Disagreements were resolved by discussion between reviewers. We extracted relevant data on study characteristics, process and clinical outcomes in a structured excel sheet. We evaluated methodological quality using the Cochrane Risk of Bias tool for RCTs [25] and the Risk Of Bias In Non-randomised Studies of interventions for non-randomised studies [26].

As anticipated, we were not able to conduct meta-analyses, due to the significant methodological and clinical diversity, statistical heterogeneity, inconsistency, and incompleteness of outcomes reported in the included studies. Instead, we used narrative synthesis and present pertinent results of the included studies in a tabulated format. Findings are presented visually as harvest plots, which summarise the direction and significance of the effect on process and clinical outcomes for each of the studies along with information about study design, study population and methodological quality $[27,28]$. To interpret the overall findings, we prioritised differences in clinical outcomes over process outcomes.

Results

Survey results: Aim 1

Survey 1: mild T2 asthma and severe T2 asthma

Of the 784 participants who started the mild T2 questionnaire, 507 also started the severe T2 asthma questions. The majority of the participants $(n=784,70.8 \%)$ were respiratory doctors as opposed to $18.5 \%$ and $10.7 \%$ who were allergy doctors and generalists, respectively. The participants' speciality and categorisation for the sub-group analysis are summarised in table 2. Most (45.2\%) were tertiary care specialists, $32.6 \%$ and $22.2 \%$ worked in secondary or primary care respectively. 


\section{Box 1: case vignette 1}

A 22-year-old female, nonsmoker, maths student attends for a consultation in October complaining about occasional chest tightness and cough (especially when playing tennis), during late spring to mid-summer for the past 4 years. She has never used any inhalers for her chest symptoms. Regular chest auscultation provides you with normal lung sounds.

She also mentions that during the same months she has been experiencing watery eyes and nose, nasal congestion as well as sneezing. These symptoms began early at adolescence and have been managed with as-needed over the counter antihistamines. She was diagnosed with eczema and egg allergy as a toddler with both conditions having resolved by the age of 10 years, which was the age she was last evaluated in an allergy clinic. She has a cat at home.

Additional information

Chest auscultation with fierce exhalation provides normal sounds. You had the possibility of performing spirometry and received the following outcomes: baseline spirometry resulted in a forced expiratory volume in $1 \mathrm{~s}\left(\mathrm{FEV}_{1}\right) /$ forced vital capacity (FVC) ratio of 0.75 and administration of $400 \mu \mathrm{g}$ salbutamol increased $\mathrm{FEV}_{1}$ by $10 \%(150 \mathrm{~mL})$. What is your diagnosis and how would you manage the patient?

Follow-up

The patient comes back during the pollen season. She reports episodes of chest tightness and cough especially early in the morning when she is walking to work through a park and if walking back home late evening. She additionally mentions wakening up at night due to chest tightness and nasal blockage. She has been avoiding playing tennis because of these symptoms. She is receiving her antihistamine daily but no nasal spray. Regular chest auscultation provides you with normal lung sounds. Spirometry with reversibility results in a $13 \%(220 \mathrm{~mL})$ increase in $\mathrm{FEV}_{1}$ post bronchodilator administration.

TABLE 1 Systematic review (SR) questions

SR-1: Effectiveness of strategies aimed to improve adherence to guidelines on the diagnosis, assessment and long-term management of asthma Population Patients with a clinical diagnosis of asthma. Patients with a clinical suspicion of asthma, for studies evaluating asthma diagnosis. Intervention Interventions aimed to improve the adherence of clinicians to guidelines on the diagnosis, assessment and long-term management of asthma.

Comparator Any other intervention aimed to improve the adherence of clinicians to guidelines on the diagnosis, assessment and long-term management of asthma, or no intervention.

Outcomes Clinical outcomes such as frequency of acute attacks, episodes of hospitalisation, asthma symptoms, or quality of life. Process outcomes, such as adherence to specific guidelines components (e.g. prescription of inhaled corticosteroids for patients requiring maintenance treatment, or delivery of smoking cessation advice).

Types of Interventional and observational comparative studies, including RCTs, cluster RCTs, comparative observational cohort studies or before-after studies studies.

SR-2: Effectiveness of strategies aimed to improve adherence to guidelines on the diagnosis, assessment and management of acute attacks

Population Patients with a clinical diagnosis of an acute asthma attack. Patients with a clinical suspicion of acute asthma attack, for studies evaluating asthma attack diagnosis.

Intervention Interventions aimed to improve the adherence of clinicians to guidelines on the diagnosis, assessment and management of acute asthma.

Comparator Any other intervention aimed to improve the adherence of clinicians to guidelines on the diagnosis, assessment and management of acute asthma, or no intervention.

Outcomes Clinical outcomes such as need for hospital admission, duration of symptoms, treatment success or failure, need for intubation or mechanical ventilation. Process outcomes, such as adherence to specific guidelines components (e.g. prescription of oral corticosteroids for all patients with an acute attack leading to an emergency presentation or hospital admission).

Types of Interventional and observational comparative studies, including RCTs, cluster RCTs, comparative observational cohort studies or before-after studies studies.

SR-3: Process and clinical outcomes in patients managed by specialists or generalists

Population Patients with a clinical diagnosis of asthma or acute asthma attack. Patients with a clinical suspicion of asthma or acute asthma attack, for studies evaluating asthma or acute asthma attack diagnosis, respectively.

Exposure $\mathrm{A} \quad$ Management by an asthma specialist (respiratory physician or allergist).

Exposure B Management by a generalist (general practitioner or internist, not specialised in asthma).

Outcomes For studies evaluating the diagnosis, assessment or long-term management of asthma: clinical outcomes such as frequency of acute attacks, episodes of hospitalisation, asthma symptoms, or quality of life. Process outcomes, such as adherence to specific guidelines components (e.g. prescription of inhaled corticosteroids for patients requiring maintenance treatment, or delivery of smoking cessation advice).

For studies evaluating the diagnosis, assessment or management of acute asthma attacks: clinical outcomes such as need for hospital admission, duration of symptoms, treatment success or failure, need for intubation or mechanical ventilation. Process outcomes, such as adherence to specific guidelines components (e.g. prescription of oral corticosteroids for all patients with an acute attack leading to an emergency presentation or hospital admission).

Types of Interventional and observational comparative studies, including RCTs, cluster RCTs, comparative observational cohort studies or before-after studies studies.

$\mathrm{RCT}$ : randomised controlled trial. 
Responses about preferred diagnostic procedures are presented in table 3. Spirometry with reversibility was the preferred diagnostic test in all groups. Home serial peak flow measurements were significantly more popular amongst the generalists than the other groups and one-third of the respiratory doctors would undertake bronchial provocation at the initial consultation compared to one-fifth of the other two groups. Of note, auscultation of the chest during forced expiration was seen as helpful by less than $50 \%$ of the respiratory doctors and generalists. Statistically significant differences between the three groups were noted for the measurement of the exhaled nitric oxide fraction $\left(F_{\mathrm{eNO}}\right)$, blood eosinophils, total serum IgE, skin prick test, specific IgE, and chest radiographs.

The mild T2 patient had normal spirometry and no bronchodilator reversibility when examined in autumn. The majority of the participants agreed that this did not exclude asthma as the patient was asymptomatic at the time. However, approximately $20 \%$ of the allergy doctors and $15 \%$ of the respiratory doctors and the generalists were "certain” about the diagnosis and would prescribe a reliever for use when needed (table e1). Note, this questionnaire was sent out in 2018, before the change in the Global Initiative for Asthma (GINA) guidelines recommending the maintenance and reliever therapy (MART) approach for mild asthma.

The majority of the participants across all groups agreed that the patient's asthma was uncontrolled (as per GINA classification) [16] when asthma status was reviewed during spring. Approximately $80 \%$ of the allergy doctors as opposed to $61.7 \%$ and $56.0 \%$ of the respiratory doctors and the generalists, respectively, replied that the patient's phenotype was allergic asthma ( $\mathrm{p}<0.0001)$. As part of the same question, $30 \%$ of the allergy doctors (additionally) included the patient under T2 asthma compared to $13.6 \%$ and $1.3 \%$ of the respiratory doctors and the generalists, respectively $(\mathrm{p}<0.0001)$ (table e1).

The majority of participants in all groups indicated that in addition to treatment for nasal symptoms, they would prescribe inhaled steroids and provide an asthma action plan. All asthma treatment options were similarly popular in the three groups except that half of the allergy doctors would commence the patient on allergen immunotherapy compared to $6.7 \%$ and $2.7 \%$, respectively, in the other groups $(\mathrm{p}<0.0001)$ (table e4).

\section{Severe T2 asthma}

\section{Box 2: case vignette 2}

A 21-year-old male (body mass index 23) comes for a consultation due to coughing, shortness of breath and wheezing. He has been suffering with asthma since childhood. From 3-12 years of age he was treated with inhaled budesonide, later on with fluticasone/salmeterol 50/250 dry powder inhaler, one puff twice-daily. While over the past 4 years he has been treated with fluticasone/salmeterol 50/500 dry powder inhaler, one puff twice-daily. Despite this treatment, he suffers from night symptoms twice a week which prompt him to use salbutamol. Playing football or cycling also cause asthma exacerbation especially during spring. He complains of itchy eyes and nose, sneezing and runny nose all year round but worse during spring. He uses loratadine on demand for his nasal and ocular symptoms.

He is a student in journalism, with no exposure to chemicals or other substances and doesn't smoke. He lives in a house with a tree garden in a small town and does not keep pets.

In the patient with severe T2 asthma, spirometry with reversibility, $F_{\mathrm{eNO}}$, blood eosinophils, total IgE, skin prick test, specific IgE, and chest radiographs were all statistically less popular among the generalists than specialists (table 3).

The majority of participants agreed that the patient's asthma was uncontrolled (as per GINA guidelines). Only $66 \%$ of the generalists versus $91.9 \%$ of the allergy doctors and $76.4 \%$ of the respiratory doctors would evaluate the presence of comorbidities in order to manage this patient $(\mathrm{p}<0.0001)$. More than $80 \%$ of participants across all groups would evaluate patient's adherence and inhaler technique (table e2).

Significantly more allergy doctors regarded the patient's asthma type as allergic asthma (71.7\%) and/or T2 asthma $(31.3 \%)$ than the other groups $(\mathrm{p}=0.007)$. Interestingly, one-fifth of generalists and one in 10 respiratory doctors stated that they did not know the patient's asthma type $(p=0.001)$. There was widespread agreement that the patient was at risk of acute attacks (table e2).

Although around only two-thirds of participants recognised uncontrolled rhinitis as a risk factor for asthma attacks, rhinitis treatment was the most popular option for asthma management, followed by montelukast. Significant differences were noted in terms of the third most popular treatment choice which was 
TABLE 2 Healthcare profession/level of training and subsequent categorisation in the analyses of the survey

Category

1st survey: mild type 2 and severe type 2 asthma

Allergy-asthma specialist

Allergy specialist

Trainee in allergy

Respiratory-asthma specialist

Respiratory doctors

Trainee in respiratory medicine

General practitioner

Internist

Specialist nurse

Trainee general practitioner

Trainee in internal medicine

Nurse trainee

2nd survey: non-type 2 asthma

Allergy-asthma specialist

Allergy specialist

Trainee in allergy

Respiratory-asthma specialist

Respiratory doctors

Trainee in respiratory medicine

General practitioner

Internist

Specialist nurse

Trainee general practitioner

Trainee in internal medicine

Nurse trainee n (\%)

Categories in the analyses

\begin{tabular}{rr}
$22(2.5)$ & Allergy doctor \\
$133(15.2)$ & Allergy doctor \\
$9(1.0)$ & Allergy doctor \\
$123(14.1)$ & Respiratory doctor \\
$456(52.1)$ & Respiratory doctor \\
$34(3.9)$ & Respiratory doctor \\
$48(5.5)$ & Generalist \\
$28(3.2)$ & Generalist \\
$13(1.5)$ & Generalist \\
$4(0.5)$ & Generalist \\
$4(0.5)$ & Generalist \\
$1(0.1)$ & Generalist \\
$30(4.4)$ & Allergy doctor \\
$163(24.0)$ & Allergy doctor \\
$12(1.8)$ & Allergy doctor \\
$80(11.8)$ & Respiratory doctor \\
$245(36.1)$ & Respiratory doctor \\
$13(1.9)$ & Respiratory doctor \\
$99(14.6)$ & Generalist \\
$16(2.4)$ & Generalist \\
$14(2.1)$ & Generalist \\
$4(0.6)$ & Generalist \\
$2(0.3)$ & Generalist \\
$1(0.2)$ & Generalist \\
\hline & \\
&
\end{tabular}

tiotropium for the respiratory doctors $(46.5 \%, \mathrm{p}<0.0001)$ and allergen immunotherapy for the allergy doctors. (50.5\%, $\mathrm{p}<0.0001)$ (table e2).

The majority of participants would proceed with an asthma control test and/or a lung function with reversibility test at the patient's follow-up appointment. Fewer (53.2\%) generalists would use $F_{\text {eNo }}$ to investigate asthma control compared to allergy doctors (73.7\%) and respiratory doctors (69.5\%) ( $\mathrm{p}=0.04)$. If asthma control was not achieved, $40 \%$ of generalists would refer the patient to an asthma clinic while most of the allergy and respiratory doctors would start the patient on omalizumab (table e2).

Survey 2: non T2 asthma

Box 3: case vignette 3

A 50-year-old female attends as an emergency due to breathlessness. She reports that her dyspnoea has worsened over the last 2 weeks despite using two puffs of beclomethasone dipropionate/formoterol (100/6 $\mu \mathrm{g})$ twice daily and that she now needs to use her reliever (salbutamol) four times a day. On presentation, she talks in phrases but wheezes, her oxygen saturation is $92 \%$, pulse rate is 118 beats per min, respiratory rate is 28 breaths per min, $\mathrm{FEV}_{1} 72 \%$ pred, $\mathrm{FVC} 82 \%$ pred, $\mathrm{FEV}_{1} / \mathrm{FVC} 0.68$ while electrocardiography is unremarkable. She was diagnosed with asthma 10 years ago (provocative concentration causing a $20 \%$ fall in $\mathrm{FEV}_{1}$ for methacholine $<4 \mathrm{mg} \cdot \mathrm{mL}^{-1}$ ), skin prick testing to common aeroallergens was negative. Since then she has been on high doses of ICS but often uses salbutamol after exercise and sometimes during the night. She has to take oral corticosteroids around four times a year for asthma exacerbations and was hospitalised due to asthma twice in the past 5 years (once in the intensive care unit). She is $160 \mathrm{~cm}$ tall, weighs $90 \mathrm{~kg}$, works in a dye factory and has been occasionally smoking the past 30 years.

Follow-up

Spirometry results are as follows: $\mathrm{FEV}_{1} 79 \%$ pred, FVC $82 \%$ pred, $\mathrm{FEV}_{1} / \mathrm{FVC} 0.72$, reversibility $7 \%$ ( $150 \mathrm{~mL}$ ). Chest auscultation normal. She still needs to use her reliever at least three times a week.

$F_{\mathrm{eNO}}$ is $6 \mathrm{ppb}$. Skin prick testing with common aeroallergens is negative. Blood eosinophils are $48 \mathrm{cells} \cdot \mathrm{LL}^{-1}$.

The majority (49.9\%) of the 677 participants were respiratory doctors as opposed to $30.3 \%$ and $19.8 \%$ who were allergy doctors and generalists, respectively (table 2). Most (45\%) worked in tertiary care, while approximately $26 \%$ and $29 \%$ were working in secondary and primary care, respectively. 
TABLE 3 Preferred diagnostic procedure in different subtypes of asthma as reported in the online survey

\begin{tabular}{|c|c|c|c|c|}
\hline & Allergy doctors (\%) & Respiratory doctors (\%) & Generalists (\%) & p-value \\
\hline \multicolumn{5}{|l|}{ Mild type 2 asthma } \\
\hline Spirometry with reversibility test & 95.0 & 96.4 & 86.9 & 0.001 \\
\hline Peak flow & 24.1 & 27.8 & 39.3 & 0.04 \\
\hline$F_{\mathrm{eNO}}$ & 49.0 & 58.7 & 41.7 & $<0.0001$ \\
\hline Blood eosinophils & 57.2 & 73.7 & 63.1 & $<0.0001$ \\
\hline Total IgE & 49.7 & 63.6 & 41.7 & 0.006 \\
\hline Skin prick test & 93.1 & 65.4 & 50.0 & $<0.0001$ \\
\hline Specific IgE & 53.1 & 38.0 & 32.1 & 0.001 \\
\hline Chest radiograph & 36.6 & 55.7 & 23.8 & $<0.0001$ \\
\hline ENT examination & 31.7 & 31.4 & 29.8 & 0.95 \\
\hline Bronchoscopy & 0 & 2.5 & 1.2 & 0.12 \\
\hline Bronchial provocation & 19.3 & 31.9 & 20.2 & 0.002 \\
\hline Bacterial culture & 4.1 & 7.4 & 7.1 & 0.38 \\
\hline Detailed history & 70.3 & 68.1 & 66.7 & 0.82 \\
\hline Chest auscultation & 55.9 & 48.3 & 41.7 & 0.10 \\
\hline Serial peak flow & 53.1 & 62.9 & 75.0 & 0.004 \\
\hline \multicolumn{5}{|l|}{ Severe type 2 asthma } \\
\hline Spirometry with reversibility test & 98.0 & 96.4 & 85.1 & 0.001 \\
\hline Peak flow & 19.2 & 24.1 & 25.5 & 0.55 \\
\hline$F_{\mathrm{eNO}}$ & 74.8 & 79.9 & 48.9 & 0.004 \\
\hline Blood eosinophils & 79.8 & 85.9 & 68.1 & 0.006 \\
\hline Total IgE & 60.6 & 77.6 & 36.2 & $<0.0001$ \\
\hline Skin prick test & 99.0 & 78.4 & 57.4 & $<0.0001$ \\
\hline Specific IgE & 55.6 & 41.0 & 34.0 & 0.01 \\
\hline Chest radiograph & 39.4 & 59.8 & 27.7 & $<0.0001$ \\
\hline ENT examination & 40.4 & 34.6 & 27.7 & 0.30 \\
\hline Bronchoscopy & 1.0 & 1.7 & 2.1 & 0.86 \\
\hline Bronchial provocation & 8.0 & 10.8 & 4.3 & 0.30 \\
\hline Bacterial culture & 9.1 & 8.6 & 8.5 & 0.99 \\
\hline Detailed history & 78.8 & 79.5 & 80.8 & 0.96 \\
\hline Chest auscultation & 83.8 & 81.7 & 76.6 & 0.57 \\
\hline Serial peak flow & 37.4 & 41.3 & 48.9 & 0.42 \\
\hline Check prescriptions & 76.8 & 85.3 & 83.0 & 0.13 \\
\hline Assess inhalation technique & 92.9 & 91.7 & 85.1 & 0.26 \\
\hline \multicolumn{5}{|l|}{ Non-type 2 asthma } \\
\hline Spirometry with reversibility test & 65.4 & 69.5 & 49.2 & $<0.0001$ \\
\hline Peak flow & 14.6 & 21.0 & 28.4 & 0.009 \\
\hline$F_{\mathrm{eNO}}$ & 50.2 & 49.7 & 26.9 & $<0.0001$ \\
\hline Blood eosinophils & 53.2 & 61.2 & 38.1 & $<0.0001$ \\
\hline Total IgE & 44.9 & 479 & 19.4 & $<0.0001$ \\
\hline Skin prick test & 26.3 & 14.2 & 9.0 & $<0.0001$ \\
\hline Specific lgE & 22.4 & 25.2 & 11.9 & 0.007 \\
\hline Chest radiograph & 49.30 & 55.9 & 30.6 & $<0.0001$ \\
\hline ENT examination & 30.2 & 23.1 & 11.2 & $<0.0001$ \\
\hline Bronchoscopy & 1.5 & 3.2 & 2.2 & 0.42 \\
\hline Bronchial provocation & 1.5 & 4.1 & 2.2 & 0.17 \\
\hline Bacterial culture & $17 . .1$ & 17.8 & 4.5 & 0.001 \\
\hline Detailed history & 65.8 & 67.8 & 53.7 & 0.01 \\
\hline Chest auscultation & 68.8 & 71.2 & 61.2 & 0.12 \\
\hline Occupational evaluation & 55.1 & 66.3 & 56.0 & 0.02 \\
\hline Check adherence & 66.3 & 71.0 & 59.7 & 0.06 \\
\hline Assess inhaler technique & 72.2 & 79.9 & 64.9 & 0.002 \\
\hline
\end{tabular}

$F_{\mathrm{eNO}}$ : exhaled nitric oxide fraction; Ig: immunoglobulin; ENT: ear, nose and throat. \#: refers to comparisons among the three groups, using the Chi-squared test.

Deciding on emergency management was challenging for all groups and there were statistically significant differences in how much prednisolone should be prescribed (table e3). At follow-up, the priority for all groups was to ensure that inhaler technique was correct. Of note, less than two-thirds of the participants 
across all groups considered evaluating for occupational exposure in this patient who worked in a dye factory (table 3).

The majority of the participants agreed that the patient's asthma was uncontrolled and most considered that the patient's asthma phenotype was obesity related $(\mathrm{p}=0.006)$ while a significantly higher percentage (19\%) of the respiratory doctors classified the patient's asthma as T2 compared to the other specialties $(\mathrm{p}=0.002)$. Tiotropium $(\mathrm{p}=0.02)$ and education $(\mathrm{p}=0.96)$ were the most popular answers regarding the optimal long-term management of this patient. Allergy doctors were more likely to consider anti-IL-5 $(\mathrm{p}<0.0001)$ or anti-IgE $(\mathrm{p}=0.008)$ treatment (table $\mathrm{e})$.

Fewer generalists prioritised the assessment of comorbidities $(\mathrm{p}=0.049)$, adherence $(\mathrm{p}=0.01)$ and inhalation technique $(\mathrm{p}=0.05)$ compared to the other two groups. Smoking cessation was prioritised by all groups but pulmonary rehabilitation was chosen more often by respiratory doctors and generalists than allergy doctors (table e3).

\section{Systematic review results: Aims 2 and 3}

Details of the search and selection process are summarised in a PRISMA flowchart (figure 1). Our search yielded 3722 unique titles, of which 52 studies evaluated strategies aimed at improving adherence to guidelines on diagnosis, assessment and/or long-term management of asthma, while 24 evaluated adherence to guideline recommendations on the assessment and management of acute asthma attacks. Differences in the care provided and asthma-related outcomes of patients managed by a specialist (respiratory physician or allergist), or a generalist (internist or general practitioner) were evaluated in 16 studies, of which 13 focused on long-term asthma management and three on acute attacks.

\section{Risk of bias}

Most studies evaluating strategies to improve implementation of guideline recommendations were at high/ serious risk of bias (table e4). Entirely appropriately, given that the implementation strategies were targeted at improving guideline adherence by clinical teams, all the included interventional trials were cluster randomised and therefore potentially at risk of selection and detection bias. Moreover, several trials did not evaluate asthma-related outcomes and it was not always clear if this represented reporting bias. Moderate or serious risk of bias was also identified for most observational studies, due to confounding, participant selection, and often outcome selection as well. Only one longitudinal evaluation of the primary care practices in Bavaria was deemed to be at low risk of bias (table e4).

High risk of methodological bias was identified in all 16 studies comparing care provision by specialists and generalists apart from two observational studies that were deemed of low risk (table e4). The two RCTs were at high risk of selection and detection bias, while there were concerns regarding unaddressed confounding for most of the included observational studies (specifically confounding because specialists tended to care for patients with more severe/uncontrolled asthma, and more severe acute attacks than generalists).

Strategies to improve adherence to guideline recommendations for long-term management of asthma: Aim 2

We identified 27 RCTs or cluster RCTs, 19 before-after studies, and six parallel comparative cohort studies, evaluating strategies for improving adherence to asthma guidelines (figure 2, tables 4 and e5). All but three studies were conducted in primary care settings. Specific interventions included the provision of additional clinical input by a specialist HCP (usually a specialist nurse or pharmacist; $n=13$ studies) [2940], medical education $(n=12)$ [41-52], computer decision-support systems $(n=7)$ [53-59], introduction of asthma care pathways $(n=4)$ [60-63], new local or national guideline $(n=4)$ [64-67], or the participation of the centre in asthma-related clinical trials $(n=1)$ [68]. Multifaceted quality improvement implementation strategies were evaluated in 11 studies [50, 69-79].

Process outcomes were evaluated in most studies (46 (88.5\%) out of 52), of which 33 (71.7\%) demonstrated improved adherence to guideline recommendations. The impact on asthma-related outcomes was evaluated in 31 (59.6\%) out of 52 studies. Only 18 (58.1\%) out of 31 studies showed any clinical benefit. Of note, this evaluation included the only observational study at low risk of bias, a large ( $\mathrm{n}=109$ 042 patients) multifaceted quality improvement initiative conducted in Bavarian primary care [72].

Findings stratified by the type of intervention are summarised in figure 2 and table e5. The introduction of additional specialised HCPs support for patient care (such as a respiratory trained nurse or a pharmacist) into the primary setting was evaluated in 13 studies including large cluster RCTs of high risk of bias and 

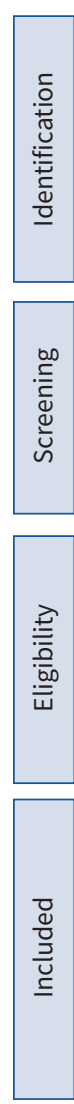

3722 records were identified

through database searching

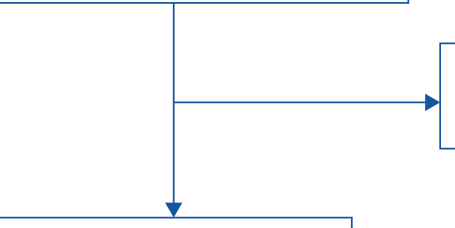

65 additional potentially eligible studies identified through reference review of the included studies title/abstract screening:

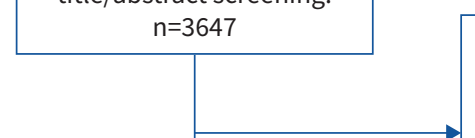

Full-text articles excluded, with reasons: $n=48$

Wrong populations: $\mathrm{n}=5$

Wrong outcomes: $\mathrm{n}=36$

Wrong interventions: $n=5$

Multiple reports of the same study without any additional relevant data identified: $\mathrm{n}=2$

FIGURE 1 PRISMA flow diagram.

TABLE 4 Types of studies evaluating the adherence to asthma guidelines and the proportion of studies demonstrating beneficial clinical and adherence outcomes among the studies evaluating such outcomes

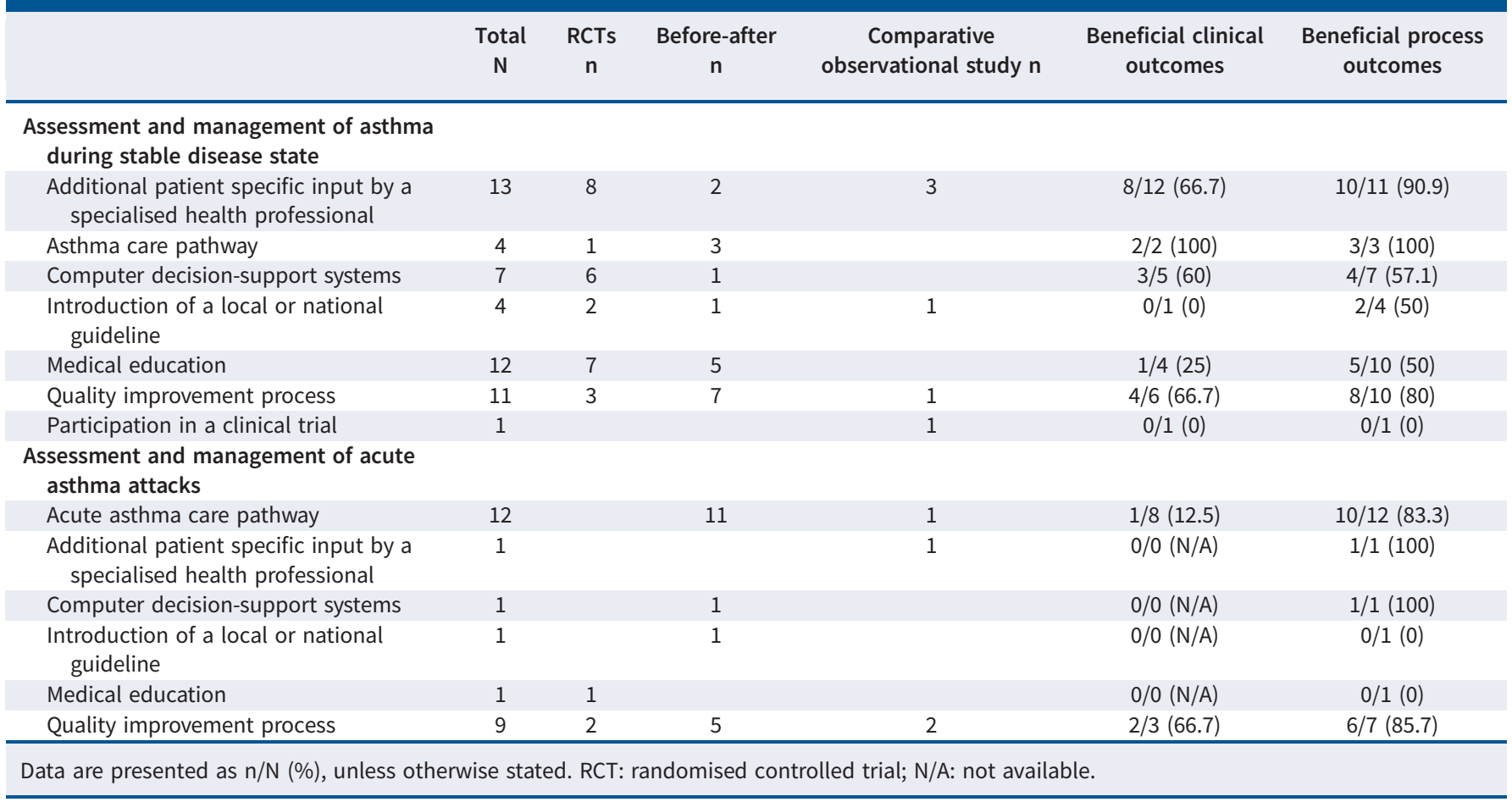




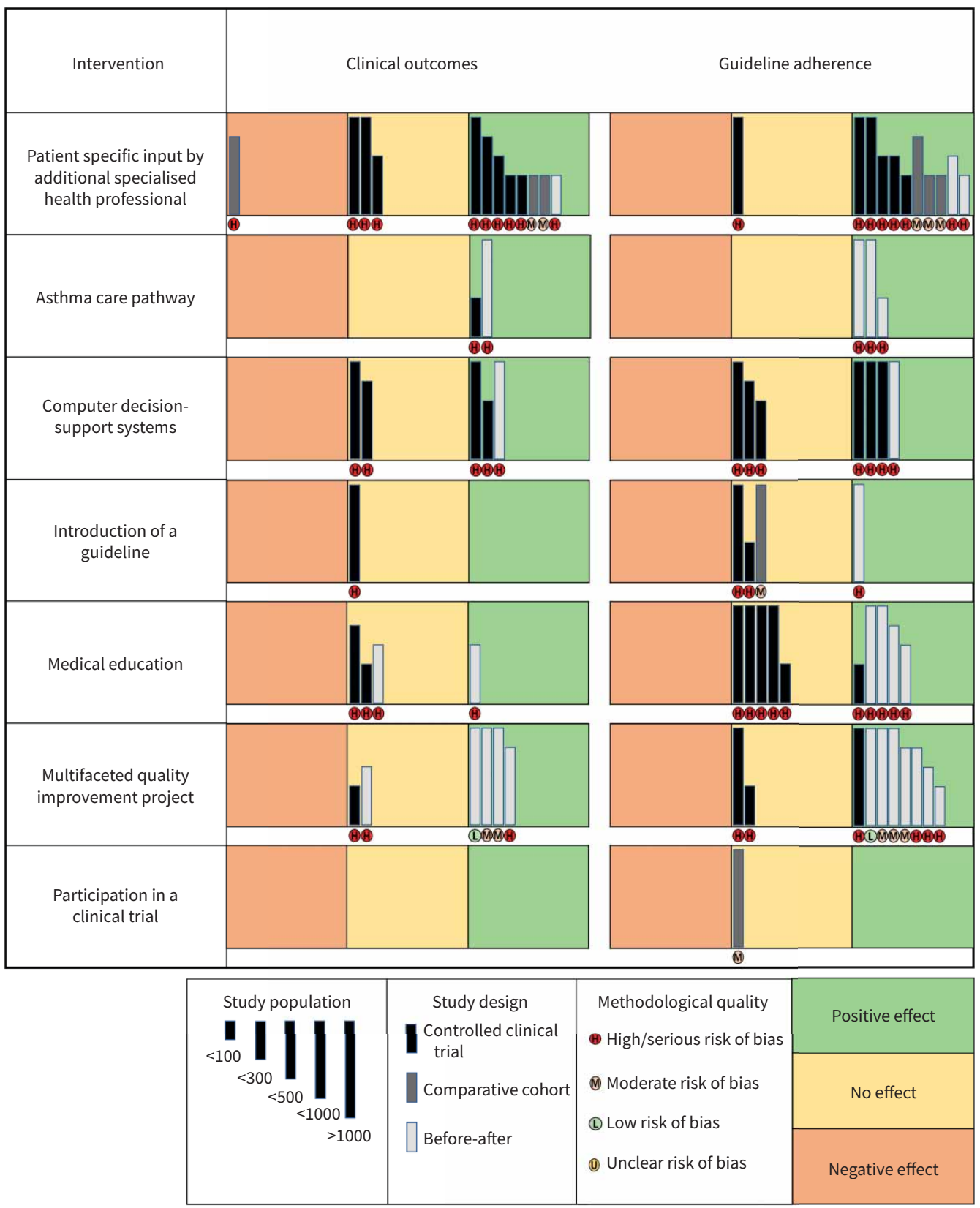

FIGURE 2 Harvest plot summarising the findings of studies evaluating interventions to improve guidelines adherence for asthma assessment and maintenance management.

observational studies that were deemed at moderate risk of bias. Most studies demonstrated improvement in process outcomes and many also demonstrated clinical benefits.

Multifaceted quality improvement projects were assessed by 11 studies including three cluster RCTs, that were of high risk of bias, and several before-after studies, including four that were deemed low or moderate risk of methodological bias. Process and clinical benefits were demonstrated in most cases, including all the low and moderate risk of bias studies. However, it should be noted that two of the three cluster RCTs did not show process benefits and the only RCT evaluating clinical outcomes did not demonstrate any benefit either.

A number of studies evaluated specific strategies for improving guideline adherence, such as computer decision-support systems, medical education, and asthma care pathways, with some promising results 
though typically in studies which combined several interventions. For example, introduction of an asthma care pathway or computer decision-support system were more effective when paired with an educational component. The introduction of new guidelines with or without a training component appeared the least effective method for improving adherence. Use of interactive and case-based learning methods appeared more effective than simple lectures or printed training material.

\section{Strategies to improve adherence to guidelines on the assessment and management of acute asthma} attacks: Aim 2

Three of the eligible studies were cluster RCTs, 17 were before-after and four were comparative cohort studies with concurrent and/or historical controls (figure 3, tables 1 and e6). Three of the included studies were conducted in primary care, while the remainder were conducted in a hospital setting (mostly in emergency departments). Specific interventions included the introduction of acute asthma care pathways $(n=12)$ [80-91], additional patient specific input by a specialised health professional $(n=1)$ [92], a computer decision-support system $(n=1)$ [93], a national clinical guideline $(n=1)$ [94], or the provision of medical education $(n=1)$ [51]. Nine studies (including the two RCTs) evaluated multi-faceted quality improvement initiatives [95-103].

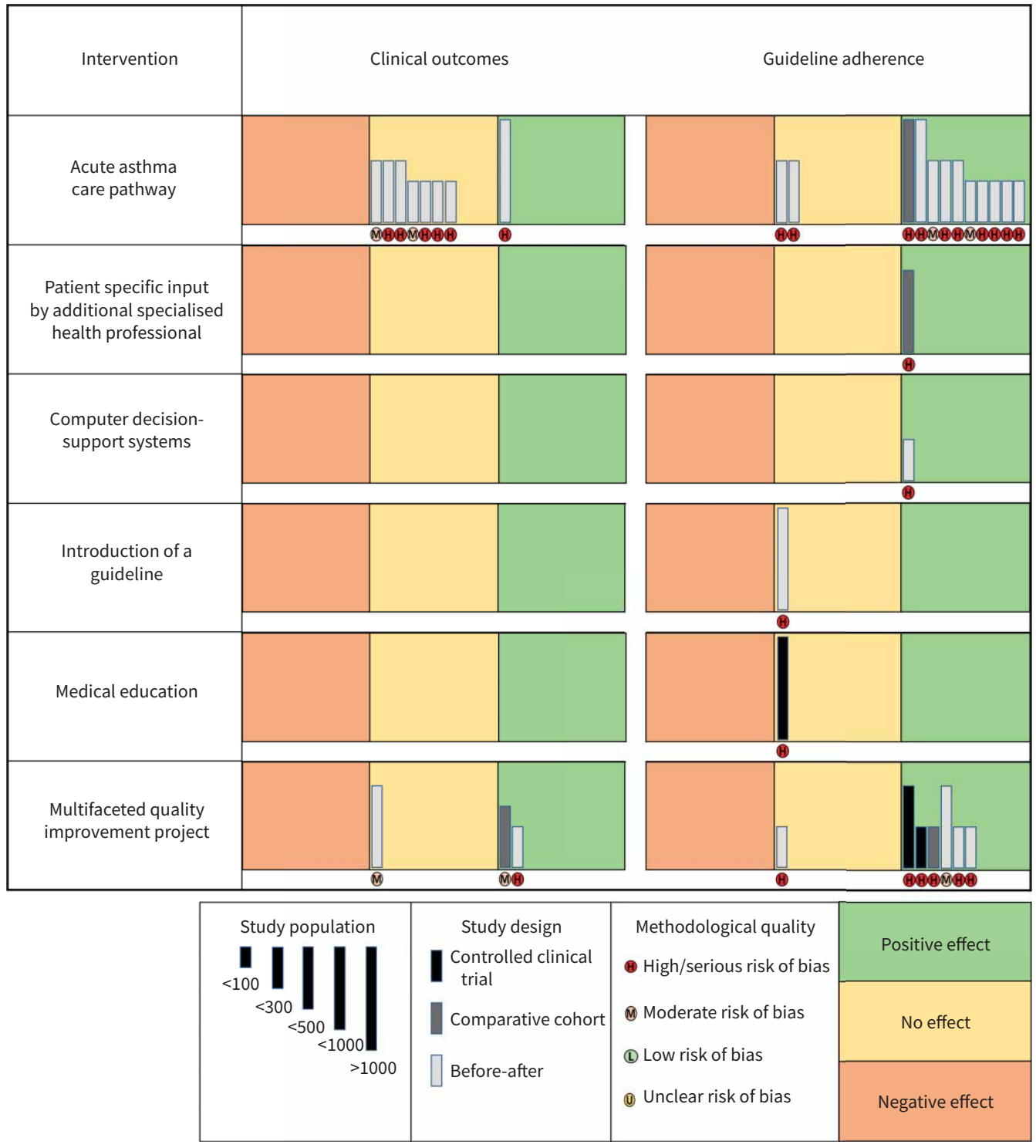

FIGURE 3 Harvest plot summarising the findings of studies evaluating interventions to improve guidelines adherence for acute asthma attacks assessment and management. 


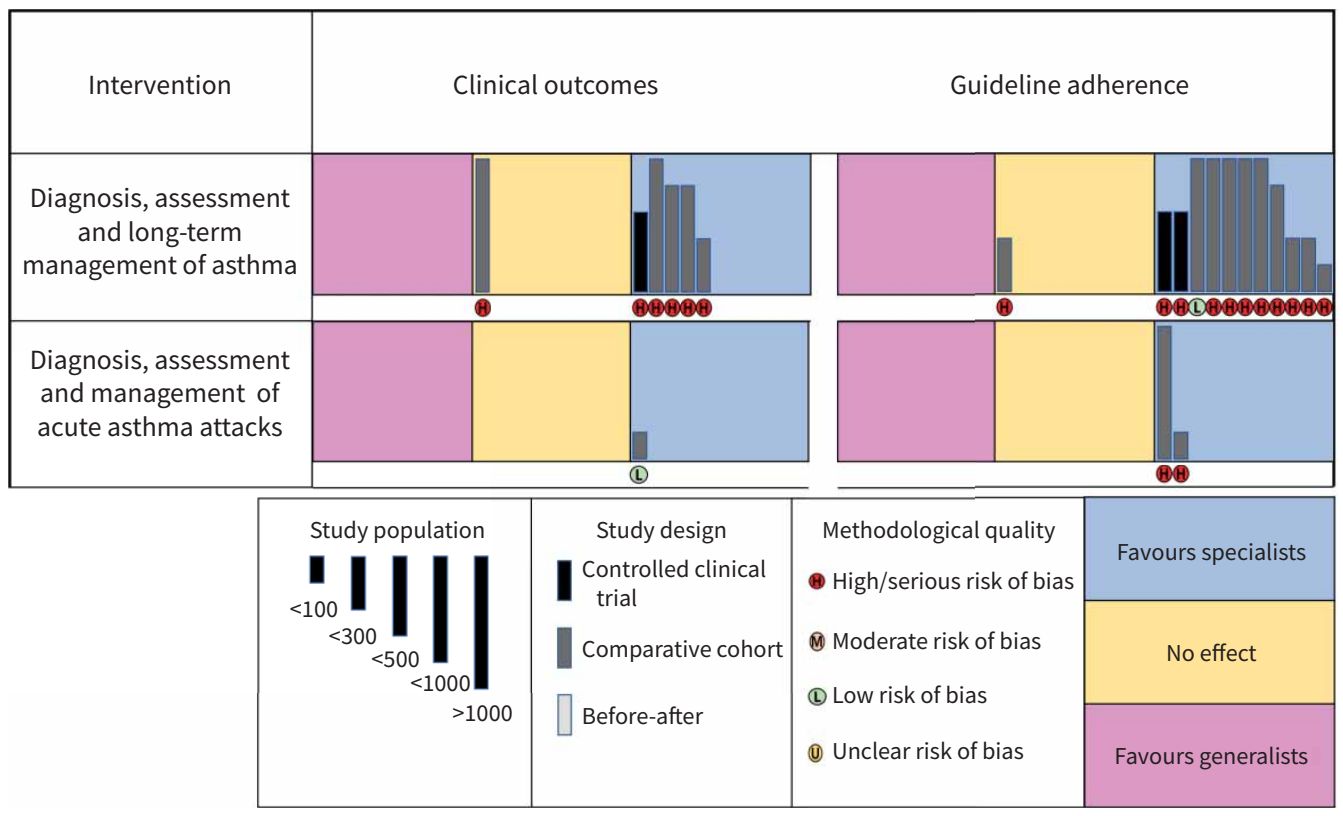

FIGURE 4 Harvest plot summarising the findings of studies evaluating differences in the adherence to asthma guidelines by specialists or generalists.

Process outcomes were evaluated in all but one study (23 (95.8\%) out of 24,), and 18 (78.3\%) out of 23 showed a beneficial impact on adherence to treatment recommendations. Clinical outcomes were evaluated in 11 (45.8\%) studies, and a clinical benefit was evident in only three (27.3\%) of them.

Acute asthma care pathways were evaluated in eight observational studies. All were deemed high risk of bias except for two that were moderate. Overall, asthma care pathways appeared effective in improving process but not clinical outcomes. Multifaceted quality improvement processes, evaluated in two cluster RCTs and six observational studies, including two that were at moderate risk of bias, showed beneficial effect on process, and possibly on clinical outcomes. Data about the clinical effectiveness of other interventions were not reported.

Differences in process and clinical outcomes of patients managed by a specialist or a generalist: Aim 3 Diagnosis, assessment and/or management of long-term asthma by specialists (respiratory physicians or allergists) compared to generalists (general physicians or general practitioners) was evaluated in two RCTs (both at high risk of bias) totalling 617 participants [104, 105], and 14 observational studies, including six large studies using routine health databases (three cross-sectional and three longitudinal studies) [106-111], and smaller cross-sectional studies, including audits (figure 4, table e7) [112-116]. Management of acute asthma attacks was evaluated in three audits, totalling 1838 participants [117-119].

Adherence to guideline recommendations was evaluated in 10 out of 12 studies, showing significantly better adherence by specialists, both for long-term asthma management and acute asthma attacks. Four out of five studies showed that specialists' care was associated with improved clinical outcomes including one cross-sectional study at low risk-of-bias which demonstrated differences in specialist/general practitioner diagnosis.

\section{Discussion}

\section{Summary and interpretation of results}

Aim 1: adherence to international asthma guidelines by HCPs of different specialties

The three online questionnaires gathered a good sample of approximately 1500 international participations in total spanning primary, secondary and tertiary care. These diverse settings clearly influenced responses despite participants being advised that they had access to all diagnostic and management facilities. For example, diagnostically, generalists favoured serial home peak flows to test for flow variability, whereas respiratory and allergy doctors would request $F_{\text {eNO }}$ which reflects familiarity and the context of their practice. Similarly, allergy doctors were confident in identifying T2 and non-T2 phenotypes, a distinction 
which appeared to have little relevance for respiratory doctors or generalists, despite the increasing recognition of disease heterogeneity [120]. However, possible differences in the terminology used across the respondents' group may also be the cause of the latter observation; characteristically, the terms used in severe asthma guidelines are eosinophilic and non-eosinophilic asthma [121, 122].

Guidelines recognise both the importance of assessing characteristic symptom patterns and undertaking objective tests in order to make a diagnosis of asthma [16, 17]. The poor sensitivity and specificity of many investigations [16, 17] was reflected in the "certainty" with which participants (in all groups) diagnosed the mild T2 patient as having asthma and offering treatment despite normal spirometry and no significant bronchodilator reversibility. Concerningly, in the severe cases, far from all participants would check the patient for comorbidities (ranging from 66\% to 93.4\%).

There was general agreement on core management strategies (role of intranasal corticosteroids, action plans, checking inhaler technique and adherence, supporting smoking cessation, treatment of nasal symptoms) but the clinical context of respondents influenced selection of other treatment modalities. For example, allergy doctors prioritised immunotherapy or biologicals, while tiotropium and pulmonary rehabilitation was chosen more often by respiratory doctors and generalists. The importance of oral steroids in an acute attack was not in doubt, but the dosages chosen varied considerably (from $1 \mathrm{mg} \cdot \mathrm{kg}^{-1}$ to $1 \mathrm{mg} \cdot \mathrm{kg}^{-1} \cdot$ day $^{-1}$ and $50 \mathrm{mg}$ prednisolone). For adults, GINA guidelines currently recommend $1 \mathrm{mg} \cdot \mathrm{kg}^{-1} \cdot$ day $^{-1}$ and up to $50 \mathrm{mg}$ per day of prednisolone or equivalent for 5-7 days [16].

GINA highlights the need to adapt asthma management strategies to enable implementation within local/ national healthcare settings [16]. Whilst some of the discrepancies identified in our survey may be due to poor dissemination or lack of knowledge, a considerable proportion of the diverse responses from allergy/ respiratory doctors and generalists are likely to reflect adaptations consistent with their different clinical settings. Effective implementation strategies are considered in the evidence from the systematic reviews.

Aim 2: effectiveness of strategies to improve implementation of guideline-recommended interventions Our systematic reviews evaluated various strategies for improving implementation of asthma guidelines. The strategies were grouped into broad categories; however, inconsistencies were observed in the results of studies evaluating strategies in each category, complicating interpretation. The main sources of heterogeneity were differences in the characteristics of individual interventions, in the methods for delivering the intervention (e.g. engagement and training of the clinical staff), the context in which the interventions were delivered and the outcomes assessed.

Patient-specific input by additional specialised HCPs was evaluated in 13 studies, including large cluster RCTs of high risk of bias and observational studies that were deemed at moderate risk of bias. The vast majority of studies evaluating this intervention demonstrated improved process outcomes and most also demonstrated clinical benefits. However, cost-effectiveness of this approach has not been evaluated, and it is not clear if this benefit is sustained after the trial is completed in case the additional support is withdrawn. In contrast, a large-scale cluster RCT in which existing primary care staff were upskilled was not effective [36].

Multicomponent quality improvement initiatives incorporating a range of implementation strategies addressing multiple challenges to guideline adherence (such as training HCPs, on-going audit and feedback/benchmarking, introduction of asthma care pathways, identification and resolution of organisational barriers [123]) appeared the most effective. Characteristically, the strategies employed in the three studies that did not show improved outcomes (either clinical or process) only included two components; audit and feedback to clinicians. Similarly, findings from studies evaluating a single intervention were in general less consistent. Multifaceted quality improvement projects incorporating a range of implementation strategies addressing challenges to guideline adherence at the level of the patient, HCP and health system were more likely to be effective. This reflects recognition of the need to take a whole systems’ approach to improving practice [124, 125].

Asthma care pathways were mostly evaluated in high-risk of bias studies, which showed clinical and process benefits. Studies evaluating other interventions were mostly at high risk of bias and their findings were either inconsistent (computer decision-support systems, medical education), or negative (introduction of a guideline, participation in clinical trials).

Some studies with longer observation periods [96, 102] noted that the impact of the interventions tended to wane and needed continuous reinforcement, for example through audit, feedback and re-training. 
Strategies for improving adherence to guidelines have been evaluated in previous systematic reviews, with consistent findings. Two systematic reviews assessing a broad range of strategies concluded that multifaceted quality improvement programmes were more effective than single component interventions, especially those based explicitly on a theoretical framework, with a strong educational component including a combination of instructional modalities, longer duration [126], and those promoting engagement at the level of the patient, HCPs and organisation [127]. Other systematic reviews focusing on specific approaches concluded that input by pharmacists [128] and asthma care protocols [129] could be beneficial, while medical education [130] and computer decision-support systems [131] were not effective, though it was not clear whether limitations of the interventions or implementation methods were responsible for this lack of observed benefit.

Aim 3: comparison of process and clinical outcomes in patients managed by specialists or generalists This systematic review was informed by fewer studies, most of which were observational and at high risk of bias. Almost all studies showed that specialist care was associated with better adherence to guideline recommendations, with some suggestion in six out of the seven studies evaluating clinical outcomes these may also be improved. It should be noted that specific findings from some of the older studies' may no longer be applicable. For example, two of these studies date from the early days of ICS prescribing when generalists may have been more cautious $[104,109]$. Improved diagnosis by specialists in a cross-sectional study at low risk of bias, might reflect better access to investigations [118]. However, specialists care was consistently associated with better outcomes in more recent studies. It should also be highlighted that only one extensive observational study evaluating process outcomes and a smaller observational study evaluating clinical outcomes were at low risk of bias, with the remaining being deemed high risk.

Asthma diagnosis, assessment and management are complex and the respective guidelines are updated frequently, making it more challenging for the generalist to keep updated. Robust, continuous, multifaceted quality improvement projects will be required to ensure that patients receive high-quality care with locally agreed referral pathways for specialists’ advice.

\section{Strengths and limitations}

The survey results provided an insight into asthma management at an international level with a good number of responses across all levels of care. A limitation to our results is that the second survey participants were not asked whether they had also taken part in the first survey, hence we cannot be sure of the total number of unique participants. Furthermore, the setup of the surveys did not facilitate analysis of the results according to the country in which the participants practised, and we are unable to establish whether variations in the answers received may have been country related. Finally, a higher proportion of the participants were respiratory physicians. However, all surveys included adequate responses from allergists and generalists, that allowed the panel to derive informed conclusions.

Our systematic reviews have a number of limitations. The study protocol was not made publicly available, however, it was developed prospectively and submitted to the ERS and EAACI. Most of the included studies were at high risk of bias, which reduces the confidence in the findings. Most included trials were cluster RCTs. Although this is the optimal study design for evaluating implementation targeted at clinical teams, they are at high risk of selection, performance and detection bias based on the Cochrane Risk of Bias tool. Confounding was the main source of bias in observational studies and despite several studies accounting for confounding factors, adjustments were not deemed adequate in most cases. In the systematic review comparing the outcomes of patients evaluated by specialists versus generalists, a key confounder was that specialists tend to care for people with more severe or uncontrolled asthma. Better outcomes among these patients could either reflect better quality of care provided by specialists, or that there was greater capacity for improvement. We were not able to conduct meta-analyses, due to the considerable clinical and methodological heterogeneity, but our results are presented in detail, both tabulated and illustrated in harvest plots to facilitate interpretation.

Last, but not least, there is significant heterogeneity among the current international asthma guidelines, thus this might be reflected in the interventions meant to improve adherence.

\section{Implications for practice and research}

Asthma is a heterogeneous disease, meaning that its diagnosis, assessment and management are complex $[16,17]$. In parallel, it is the focus of intensive research that leads to continuous change to clinical practice guidelines and practice, increasingly incorporating precision medicine interventions [21, 132]. As a result, implementation of asthma guidelines and delivery of high-quality, evidence-based medicine is challenging and often suboptimal [133-135]. Our findings suggest that continuous multifaceted quality improvement 
processes can enhance adherence to guidelines. Additional input by a specialist, either a respiratory doctor, allergist or a respiratory trained nurse or pharmacist, also appears to improve guidelines adherence and clinical outcomes, although further data is needed to confirm sustainability of these findings. Moreover, the feasibility and cost-effectiveness of these approaches should be evaluated.

Our survey revealed significant variability in practice, across different clinical settings, that reflects guideline adaptations in a real-life context, where different diagnostic or therapeutic options and sources are available. Guideline panels need to consider these practical differences when developing clinical recommendations, and to offer options for evidence-based practice in different clinical settings.

Systematic literature reviews also indicated a potential association of specialist care with improved process and clinical outcomes. However, more data are needed, as confidence was limited on this finding. Undoubtedly, the complexity of asthma care imposes the need for a multidisciplinary approach to the diagnosis and management of these patients. As a result, it is now widely recommended that patients with severe asthma should be managed in specialised severe asthma clinics [11, 16, 17, 121, 122]. However, the diagnosis and management of patients without severe asthma is also complex, but it is still unclear when generalist or specialist care is necessary [11, 16, 17, 121, 122]. This complicates the work of both generalists and specialists and, as suggested by our systematic review, may also impact on the clinical outcomes of individuals with asthma. Therefore, data are needed to inform standardisation in the indications for referral of patients for specialist review, that should be tailored to the balance of resources required for continuous multifaceted quality improvement processes in primary care versus the evaluation of an increased proportion of individuals with asthma in specialty clinics. In the meantime, locally agreed referral pathways to specialists are crucial both for generalists and for specialists from different disciplines who have different approaches to diagnostic uncertainty and managing patients with poorly controlled asthma.

The emergence of coronavirus disease 2019 has extensively affected the care of people with asthma, mainly by replacing physical appointments with virtual encounters, while in parallel reinforcing telemonitoring technologies [136, 137]. It is recognised that to some extent these practice changes introduced during 2020 will outlive the pandemic, as they appear effective, convenient for patients and require fewer resources [138-143]. An opportunity emerges to use these new technologies to enhance adherence to guidelines. For example, efficient methods for capturing disease characteristics in a computerusable format could facilitate patient profiling and strengthen decision support systems. Such interventions are already being evaluated in other disease areas with promising preliminary results $[144,145]$.

\section{Box 4: key messages}

Implementation of guidelines is different across different asthma management settings.

Guideline recommendations need to account for differences in resource availability across the various asthma care settings, including primary care.

Continuous multifaceted quality improvement processes can improve guidelines adherence.

Additional input from specialised health professionals could also be effective towards improving guidelines adherence. However, this is unlikely to be sustainable unless long-term funding is available. Locally agreed referral pathways to specialists are crucial both for generalists and specialists from different disciplines who have different approaches to diagnostic uncertainty and managing patients with poorly controlled asthma.

More data are needed to evaluate differences in process and clinical outcomes among patients managed by generalists or specialists and to facilitate standardisation in the indications for referral of patients for specialist review.

\section{Conclusion}

This evaluation, conducted as a joint initiative between EAACI and ERS, showed a significant gap in implementing asthma guidelines in real life. This calls for action on several fronts: 1) guideline developers should consider the heterogeneity of settings for asthma management in real life and tailor their recommendations accordingly; 2) multifaceted interventions should receive better funding to improve adherence to guidelines; and 3) validated referral pathways for uncontrolled asthma or for uncertain diagnosis should be prioritised. 
Acknowledgement: This study was conducted by a European Academy of Allergy and Clinical Immunology (TF 400531) and a European Respiratory Society (ERS TF-2015-16) Task Force and the authors would like to thank the two societies for their support. The authors would also like to thank the International Primary Care Respiratory Group and their President loanna Tsiligianni, at the time the surveys were launched, for disseminating the surveys among their membership. The authors would also like to thank Lindsey Kent for contributing to titles and abstracts screening.

This ERS/EAACl statement was endorsed by the ERS Executive Committee on 04 August 2021 and by EAACI Board of Officers on 06 April 2021.

Conflict of interest: A.G. Mathioudakis reports grants from Boehringer Ingelheim, outside the submitted work. O. Tsilochristou has nothing to disclose. I.M. Adcock has nothing to disclose. A. Bikov has nothing to disclose. L. Bjermer has nothing to disclose. E. Clini has nothing to disclose. B. Flood has nothing to disclose. F. Herth received personal fees for advisory board activities and lecture fees from Pulmonx, BTG, Olympus and Uptake, outside the submitted work. I. Horvath reports personal fees from AstraZeneca, Chiesi, Berlin-Chemie, Boehringer-Ingelheim, GSK, Teva, CSL, Novartis and Roche, outside the submitted work. O. Kalayci has nothing to disclose. N.G. Papadopoulos reports personal fees from Novartis, Nutricia, HAL, MENARINI/FAES FARMA, SANOFI, MYLAN/MEDA, BIOMAY, AstraZeneca, GSK, MSD, ASIT BIOTECH and Boehringer Ingelheim, and grants from Gerolymatos International SA and Capricare, outside the submitted work. D. Ryan reports personal fees from Boehringer Ingelheim, AstraZeneca, Regeneron, Trudell, GSK and Novartis, and non-financial support from Chiesi, outside the submitted work. S. Sanchez Garcia has nothing to disclose. J. Correia-de-Sousa reports other funding from Boheringer Ingelheim and Novartis, grants, personal fees and other from GSK and AstraZeneca, personal fees and other from Bial, non-financial support from Mundipharma, and personal fees from Sanofi, outside the submitted work. T Tonia reports acting as ERS Methodologist. H. Pinnock reports personal fees from lecture fees from Boehringer Ingelheim, outside the submitted work. I. Agache is an Associate Editor for Allergy and CTA. C. Janson reports personal fees from AstraZeneca, Boehringer Ingelheim, Chiesi, GlaxoSmithKline, and Novartis, outside the submitted work.

Support statement: Funding has been received from the National Institute for Health Research (NIHR Manchester Biomedical Research Centre (BRC)), European Academy of Allergy and Clinical Immunology (Grant: EAACI TF 400531) and the European Respiratory Society (Grant: ERS Guidelines Methodology Fellowship MTF 2015-1, ERS TF-2015-16). Funding information for this article has been deposited with the Crossref Funder Registry.

\section{References}

1 Gibson GJ, Loddenkemper R, Sibille Y, et al., eds. European Lung White Book. Sheffield, European Respiratory Society, 2013.

2 Ebmeier S, Thayabaran D, Braithwaite I, et al. Trends in international asthma mortality: analysis of data from the WHO Mortality Database from 46 countries (1993-2012). Lancet 2017; 390: 935-945.

3 Ernst P, Spitzer WO, Suissa S, et al. Risk of fatal and near-fatal asthma in relation to inhaled corticosteroid use. JAMA 1992; 268: 3462-3464.

4 Ställberg B, Lisspers K, Hasselgren M, et al. Asthma control in primary care. A comparison between 2001 and 2005. Prim Care Respir J 2009; 18: 279-286.

5 Demoly P, Annunziata K, Gubba E, et al. Repeated cross-sectional survey of patient-reported asthma control in Europe in the past 5 years. Eur Respir Rev 2012; 21: 66-74.

6 Ek A, Middelveld R, Bertilsson $\mathrm{H}$, et al. Chronic rhinosinusitis in asthma is a negative predictor of quality of life: results from the Swedish GA2LEN Survey. Allergy 2013; 68: 1314-1321.

$7 \quad$ Kallin SA, Lindberg E, Nilsson Sommar J, et al. Excessive daytime sleepiness in asthma: what are the risk factors? J Asthma 2018; 55: 844-850.

8 Janson C, Accordini S, Cazzoletti L, et al. Pharmacological treatment of asthma in a cohort of adults during a 20-year period: results from the European Community Respiratory Health Survey I, II and III. ERJ Open Research 2019; 5: 00073-02018.

9 O'Byrne PM, Jenkins C, Bateman ED. The paradoxes of asthma management: time for a new approach? Eur Respir J 2017; 50: 1701103.

10 Custovic A, Henderson J, Simpson A. Does understanding endotypes translate to better asthma management options for all? J Allergy Clin Immunol 2019; 144: 25-33.

11 Chung KF, Wenzel SE, Brozek JL, et al. International ERS/ATS guidelines on definition, evaluation and treatment of severe asthma. Eur Respir J 2014; 43: 343-373.

12 Krings JG, McGregor MC, Bacharier LB, et al. Biologics for severe asthma: treatment-specific effects are important in choosing a specific agent. J Allergy Clin Immunol Pract 2019; 7: 1379-1392.

13 Ryan D, Heatley H, Heaney LG, et al. Potential severe asthma hidden in UK primary care. J Allergy Clin Immunol Pract 2021; 9: 1612-1623. 
Normansell R, Kew KM, Mathioudakis AG. Interventions to improve inhaler technique for people with asthma. Cochrane Database Syst Rev 2017; 3: CD012286. .

Normansell R, Kew KM, Stovold E. Interventions to improve adherence to inhaled steroids for asthma. Cochrane Database Syst Rev 2017; 4: CD012226.

Eguiluz-Gracia I, Mathioudakis AG, Bartel S, et al. The need for clean air: the way air pollution and climate change affect allergic rhinitis and asthma. Allergy 2020; 75: 2170-2184. .

Global Initiative for Asthma. Pocket Guide for asthma management and prevention. 2019. https://ginasthma. org/wp-content/uploads/2019/04/GINA-2019-main-Pocket-Guide-wms.pdf

British Thoracic Society. Updated BTS/SIGN national guidelines on the management of asthma. 2019. https://www.brit-thoracic.org.uk/news/news/2019/btssign-british-guideline-on-the-management-of-asthma-2019/ Akinbami LJ, Salo PM, Cloutier MM, et al. Primary care clinician adherence with asthma guidelines: the National Asthma Survey of Physicians. J Asthma 2020; 57: 543-555.

Cloutier MM, Salo PM, Akinbami LJ, et al. Clinician agreement, self-efficacy, and adherence with the guidelines for the diagnosis and management of asthma. J Allergy Clin Immunol Pract 2018; 6: 886-886894.

Wiener-Ogilvie S, Pinnock H, Huby G, et al. Do practices comply with key recommendations of the British Asthma Guideline, and if not, why not? Prim Care Resp J 2007; 16: 369-377.

Agache I, Akdis CA. Precision medicine and phenotypes, endotypes, genotypes, regiotypes, and theratypes of allergic diseases. J Clin Invest 2019; 129: 1493-1503.

Higgins JPT, Thomas J, Chandler J, et al., eds. Cochrane Handbook for Systematic Reviews of Interventions version 5.1.0 (updated March 2011). Cochrane, 2011. www.training.cochrane.org/handbook

Brewis G. Guidelines for the management of asthma in adults. I. Chronic asthma. Br Med J 1990; 301: 651-653.

Higgins JPT, Altman DG, Sterne JAC. Assessing risk of bias in included studies. In: Higgins JPT, Green S, eds) Cochrane Handbook for Systematic Reviews of Interventions version 5.1.0 [updated March 2011]. The Cochrane Collaboration, 2011. www.handbook.cochrane.org

Wells GA, Shea B, O'Connell D, et al. The Newcastle-Ottawa Scale (NOS) for assessing the quality if nonrandomized studies in meta-analyses. www.ohri.ca/programs/clinical_epidemiology/oxford.htm Date last accessed: 18 August 2019

McKenzie JE, Brennan SE. Synthesizing and presenting findings using other methods. In: Higgins JPT, Thomas J, Chandler J, et al., eds. Cochrane Handbook for Systematic Reviews of Interventions version 6.0 (updated July 2019). Cochrane, 2019. www.training.cochrane.org/handbook

Pinnock H, Epiphaniou E, Pearce G, et al. Implementing supported self-management for asthma: a systematic review and suggested hierarchy of evidence of implementation studies. BMC Med 2015; 13: 127.

Armour C, Bosnic-Anticevich S, Brillant M, et al. Pharmacy Asthma Care Program (PACP) improves outcomes for patients in the community. Thorax 2007; 62: 496-502.

Coleman $\mathrm{Cl}$, Reddy $\mathrm{P}$, Laster-Bradley NM, et al. Effect of practitioner education on adherence to asthma treatment guidelines. Ann Pharmacother 2003; 37: 956-961.

Dickinson J, Hutton S, Atkin A. Implementing the British Thoracic Society's guidelines: the effect of a nurse-run asthma clinic on prescribed treatment in an English general practice. Respir Med 1998; 92: 264-267.

Herborg H, Soendergaard B, Jorgensen T, et al. Improving drug therapy for patients with asthma-part 2: use of antiasthma medications. J Am Pharm Assoc (Wash) 2001; 41: 551-559.

Lindberg M, Ahlner J, Ekström T, et al. Asthma nurse practice improves outcomes and reduces costs in primary health care. Scand J Caring Sci 2002; 16: 73-78.

Manfrin A, Tinelli M, Thomas T, et al. A cluster randomised control trial to evaluate the effectiveness and cost-effectiveness of the Italian medicines use review (I-MUR) for asthma patients. BMC Health Serv Res 2017; 17: 300. .

McLean W, Gillis J, Waller R. The BC community pharmacy asthma study: a study of clinical, economic and holistic outcomes influenced by an asthma care protocol provided by specially trained community pharmacists in British Columbia. Can Respir J 2003; 10: 195-202.

Pilotto LS, Smith BJ, Heard AR, et al. Trial of nurse-run asthma clinics based in general practice versus usual medical care. Respirology 2004; 9: 356-362.

Premaratne UN, Sterne JA, Marks GB, et al. Clustered randomised trial of an intervention to improve the management of asthma: Greenwich asthma study. BMJ 1999; 318: 1251-1255.

Wong LY, Chua SS, Husin AR, et al. A pharmacy management service for adults with asthma: a cluster randomised controlled trial. Fam Pract 2017; 34: 564-573.

Yanchick JK. Implementation of a drug therapy monitoring clinic in a primary-care setting. Am J Health Syst Pharm 2000; 57: Suppl. 4, S30-S34.

Zeiger RS, Schatz M, Li Q, et al. Real-time asthma outreach reduces excessive short-acting $\beta 2$-agonist use: a randomized study. J Allergy Clin Immunol Pract 2014; 2: 445-456. . 
Ables AZ, Godenick MT, Lipsitz SR. Improving family practice residents' compliance with asthma practice guidelines. Fam Med 2002; 34: 23-28.

Bachmann MO, Bateman ED, Stelmach R, et al. Effects of PACK guide training on the management of asthma and chronic obstructive pulmonary disease by primary care clinicians: a pragmatic cluster randomised controlled trial in Florianópolis. Brazil BMJ Glob Health 2019; 4: e001921.

Baldacci S, Maio S, Simoni M, et al. The ARGA study with general practitioners: impact of medical education on asthma/rhinitis management. Respir Med 2012; 106: 777-785.

Bender BG, Dickinson P, Rankin A, et al. The Colorado Asthma Toolkit Program: a practice coaching intervention from the High Plains Research Network. J Am Board Fam Med 2011; 24: 240-248.

Bender BG, Dingae MB, Fending D, et al. Respiratory care training for safety-net primary care practices. Fam Med 2015; 47: 554-557.

Cicutto L, Dingae MB, Langmack EL. Improving asthma care in rural primary care practices: a performance improvement project. J Contin Educ Health Prof 2014; 34: 205-214.

Cleland JA, Hall S, Price D, et al. An exploratory, pragmatic, cluster randomised trial of practice nurse training in the use of asthma action plans. Prim Care Respir J 2007; 16: 311-318.

Daniels EC, Bacon J, Denisio S, et al. Translation squared: improving asthma care for high-disparity populations through a safety net practice-based research network. J Asthma 2005; 42: 499-505.

Goeman DP, Sanci LA, Scharf SL, et al. Improving general practice consultations for older people with asthma: a cluster randomised control trial. Med J Aust 2009; 191: 113-117.

Greene J, Rogers VW, Yedidia MJ. The impact of implementing a chronic care residency training initiative on asthma outcomes. Acad Med 2007; 82: 161-167.

Mold JW, Fox C, Wisniewski A, et al. Implementing asthma guidelines using practice facilitation and local learning collaboratives: a randomized controlled trial. Ann Fam Med 2014; 12: 233-240.

Veninga CC, Lagerløv P, Wahlström R, et al. Evaluating an educational intervention to improve the treatment of asthma in four European countries. Drug Education Project Group. Am J Respir Crit Care Med 1999; 160: 1254-1262.

Cho SH, Jeong JW, Park HW, et al. Effectiveness of a computer-assisted asthma management program on physician adherence to guidelines. J Asthma 2010; 47: 680-686.

Eccles M, McColl E, Steen N, et al. Effect of computerised evidence based guidelines on management of asthma and angina in adults in primary care: cluster randomised controlled trial. BMJ 2002; 325: 941.

Kuilboer MM, van Wijk MA, Mosseveld M, et al. Computed critiquing integrated into daily clinical practice affects physicians' behavior - a randomized clinical trial with AsthmaCritic. Methods Inf Med 2006; 45: 447-454.

Martens JD, van der Weijden T, Severens JL, et al. The effect of computer reminders on GPs' prescribing behaviour: a cluster-randomised trial. Int J Med Inform. 2007; 76: Suppl. 3, S403-S416.

McCowan C, Neville RG, Ricketts IW, et al. Lessons from a randomized controlled trial designed to evaluate computer decision support software to improve the management of asthma. Med Inform Internet Med 2001; 26: 191-201.

Tamblyn R, Ernst P, Winslade N, et al. Evaluating the impact of an integrated computer-based decision support with person-centered analytics for the management of asthma in primary care: a randomized controlled trial. J Am Med Inform Assoc 2015; 22: 773-783.

Tierney WM, Overhage JM, Murray MD, et al. Can computer-generated evidence-based care suggestions enhance evidence-based management of asthma and chronic obstructive pulmonary disease? A randomized, controlled trial. Health Serv Res 2005; 40: 477-497.

Renzi PM, Ghezzo H, Goulet S, et al. Paper stamp checklist tool enhances asthma guidelines knowledge and implementation by primary care physicians. Can Respir J 2006; 13: 193-197.

Ruoff G. Effects of flow sheet implementation on physician performance in the management of asthmatic patients. Fam Med 2002; 34: 514-517.

To T, Cicutto L, Degani N, et al. Can a community evidence-based asthma care program improve clinical outcomes?: a longitudinal study. Med Care 2008; 46: 1257-1266.

Yawn BP, Bertram S, Wollan P. Introduction of Asthma APGAR tools improve asthma management in primary care practices. J Asthma Allergy 2008; 1: 1-10.

Baker R, Fraser RC, Stone M, et al. Randomised controlled trial of the impact of guidelines, prioritized review criteria and feedback on implementation of recommendations for angina and asthma. Br J Gen Pract 2003; 53: 284-291.

Feder G, Griffiths C, Highton C, et al. Do clinical guidelines introduced with practice based education improve care of asthmatic and diabetic patients? A randomised controlled trial in general practices in east London. BMJ 1995; 311: 1473-1478.

Kim SH, Cho BL, Shin DW, et al. The effect of asthma clinical guideline for adults on inhaled corticosteroids prescription trend: a quasi-experimental study. J Korean Med Sci 2015; 30: 1048-1054. 
Wright J, Warren E, Reeves J, et al. Effectiveness of multifaceted implementation of guidelines in primary care. J Health Serv Res Policy 2003; 8: 142-148.

Andersen M, Kragstrup J, Søndergaard J. How conducting a clinical trial affects physicians' guideline adherence and drug preferences. JAMA 2006; 295: 2759-2764.

Blais R, Laurier C, Paré M. Effect of feedback letters to physicians and pharmacists on the appropriate use of medication in the treatment of asthma. J Asthma 2008; 45: 227-231.

Jans MP, Schellevis FG, Van Hensbergen W, et al. Improving general practice care of patients with asthma or chronic obstructive pulmonary disease: evaluation of a quality system. Eff Clin Pract 2000; 3: 16-24.

Jans MP, Schellevis FG, Le Coq EM, et al. Health outcomes of asthma and COPD patients: the evaluation of a project to implement guidelines in general practice. Int J Qual Health Care 2001; 13: 17-25.

Licskai C, Sands T, Ong M, et al. Using a knowledge translation framework to implement asthma clinical practice guidelines in primary care. Int J Qual Health Care 2012; 24: 538-546.

Mehring M, Donnachie E, Mutschler R, et al. Disease management programs for patients with asthma in Germany: a longitudinal population-based study. Respir Care 2013; 58: 1170-1177.

Mohammad Y, Shaaban R, Salman HA, et al. Improving the quality of hospital care provided for asthma out-patients in a country in turmoil: a report from Syria. J Thorac Dis 2019; 11: 1047-1055.

medical group. Dis Manag 2004; 7: 102-111.

Roberts DH, Gilmartin GS, Neeman N, et al. Design and measurement of quality improvement indicators in ambulatory pulmonary care: creating a "culture of quality" in an academic pulmonary division. Chest 2009; 136: 1134-1140.

Rojanasarot S, Heins Nesvold J, Karaca-Mandic P, et al. Enhancing guideline-based asthma care processes through a multi-state, multi-center quality improvement program. J Asthma 2019; 56: 440-450.

Rojanasarot S, Carlson AM, St Peter WL, et al. Reducing potentially preventable health events among patients with asthma through multi-state, multi-center quality improvement program. J Asthma 2021; 58: 874-882.

Schneider A, Wensing M, Biessecker $\mathrm{K}$, et al. Impact of quality circles for improvement of asthma care: results of a randomized controlled trial. J Eval Clin Pract 2008; 14: 185-190.

Abisheganaden J, Chee CB, Goh SK, et al. Impact of an asthma care path on the management of acute asthma exacerbations. Ann Acad Med Singap 2001; 30: Suppl. 4, $22-26$.

Davies B, Edwards N, Ploeg J, et al. Insights about the process and impact of implementing nursing guidelines on delivery of care in hospitals and community settings. BMC Health Serv Res 2008; 8: 29.

Gentile NT, Ufberg J, Barnum M, et al. Guidelines reduce x-ray and blood gas utilization in acute asthma. Am J Emerg Med 2003; 21: 451-453.

Goldberg R, Chan L, Haley P, et al. Critical pathway for the emergency department management of acute asthma: effect on resource utilization. Ann Emerg Med 1998; 31: 562-567.

Joe $\mathrm{RH}$, Kellermann A, Arheart $\mathrm{K}$, et al. Emergency department asthma treatment protocol. Ann Pharmacother 1992; 26: 472-476.

Lougheed MD, Olajos-Clow J, Szpiro K, et al. Multicentre evaluation of an emergency department asthma care pathway for adults. CJEM 2009; 11: 215-229.

Mackey D, Myles $\mathrm{M}$, Spooner $\mathrm{CH}$, et al. Changing the process of care and practice in acute asthma in the emergency department: experience with an asthma care map in a regional hospital. CJEM 2007; 9: 353-365.

McFadden ER Jr, Elsanadi N, Dixon L, et al. Protocol therapy for acute asthma: therapeutic benefits and cost savings. Am J Med 1995; 99: 651-661.

Robinson SM, Harrison BD, Lambert MA. Effect of a preprinted form on the management of acute asthma in an accident and emergency department. J Accid Emerg Med 1996; 13: 93-97.

Rowe BH, Chahal AM, Spooner $\mathrm{CH}$, et al. Increasing the use of anti-inflammatory agents for acute asthma in the emergency department: experience with an asthma care map. Can Respir J 2008; 15: 20-26.

Steurer-Stey C, Grob U, Jung S, et al. Education and a standardized management protocol improve the assessment and management of asthma in the emergency department. Swiss Med Wkly 2005; 135: 222-227.

Sucov A, Veenema TG. Implementation of a disease-specific care plan changes clinician behaviors. Am J Emerg Med 2000; 18: 367-371.

Chew SY, Leow JYL, Chan AKW, et al. Improving asthma care with Asthma-COPD Afterhours Respiratory Nurse at Emergency (A-CARE). BMJ Open Qual 2020; 9: e000894.

Kwok R, Dinh M, Dinh $\mathrm{D}$, et al. Improving adherence to asthma clinical guidelines and discharge documentation from emergency departments: implementation of a dynamic and integrated electronic decision support system. Emerg Med Australas 2009; 21: 31-37.

94 Pearson MG, Ryland I, Harrison BD. Comparison of the process of care of acute severe asthma in adults admitted to hospital before and $1 \mathrm{yr}$ after the publication of national guidelines. Respir Med 1996; 90: 539-545. Akerman MJ, Sinert R. A successful effort to improve asthma care outcome in an inner-city emergency department. J Asthma 1999; 36: 295-303. 
Chouaid C, Bal JP, Fuhrman C, et al. Standardized protocol improves asthma management in emergency department. J Asthma 2004; 41: 19-25.

de Dalcin PT, da Rocha PM, Franciscatto E, et al. Effect of clinical pathways on the management of acute asthma in the emergency department: five years of evaluation. $J$ Asthma 2007; 44: 273-279.

Doherty SR, Jones PD. Use of an 'evidence-based implementation' strategy to implement evidence-based care of asthma into rural district hospital emergency departments. Rural Remote Health 2006; 6: 529.

Doherty SR, Jones PD, Davis L, et al. Evidence-based implementation of adult asthma guidelines in the emergency department: a controlled trial. Emerg Med Australas 2007; 19: 31-38.

asthma care. Ann Emerg Med. 1999; 34: 321-325.

Foster JM, Hoskins G, Smith B, et al. Practice development plans to improve the primary care management of acute asthma: randomised controlled trial. BMC Fam Pract 2007; 8: 23.

Pinnock H, Hoskins G, Smith B, et al. A pilot study to assess the feasibility and acceptability of undertaking acute asthma professional development in three different UK primary care settings. Prim Care Respir J 2003; 12: $7-11$.

Stell IM. Asthma management in accident and emergency and the BTS guidelines- a study of the impact of clinical audit. J Accid Emerg Med 1996; 13: 392-394.

Harmsen L, Nolte H, Backer V. The effect of generalist and specialist care on quality of life in asthma patients with and without allergic rhinitis. Int Arch Allergy Immunol 2010; 152: 288-294.

Zeiger RS, Heller S, Mellon MH, et al. Facilitated referral to asthma specialist reduces relapses in asthma emergency room visits. J Allergy Clin Immunol 1991; 87: 1160-1168.

Chou CL, Perng DW, Lin TL, et al. Analysis of prescription pattern and guideline adherence in the management of asthma among medical institutions and physician specialties in Taiwan between 2000 and 2010. Clin Ther 2015; 37: 2275-2285.

Erickson S, Tolstykh I, Selby JV, et al. The impact of allergy and pulmonary specialist care on emergency asthma utilization in a large managed care organization. Health Serv Res 2005; 40: 1443-1465.

Meng YY, Leung KM, Berkbigler D, et al. Compliance with US asthma management guidelines and specialty care: a regional variation or national concern? J Eval Clin Pract 1999; 5: 213-221.

Morishima T, Otsubo T, Gotou E, et al. Physician adherence to asthma treatment guidelines in Japan: focus on inhaled corticosteroids. J Eval Clin Pract 2013; 19: 223-229.

Vollmer WM, O'Hollaren M, Ettinger KM, et al. Specialty differences in the management of asthma. A cross-sectional assessment of allergists' patients and generalists' patients in a large HMO. Arch Intern Med 1997; 157: 1201-1208.

1 Wu AW, Young Y, Skinner EA, et al. Quality of care and outcomes of adults with asthma treated by specialists and generalists in managed care. Arch Intern Med 2001; 161: 2554-2560.

Abdulwadud OA, Abramson MJ, Light L, et al. Comparison of patients with asthma managed in general practice and in a hospital clinic. Med J Aust 1999; 171: 72-75.

Frieri M, Therattil J, Dellavecchia D, et al. A preliminary retrospective treatment and pharmacoeconomic analysis of asthma care provided by allergists, immunologists, and primary care physicians in a teaching hospital. J Asthma 2002; 39: 405-412.

4 Kanter LJ, Siegel CJ, Snyder CF, et al. Impact of respiratory symptoms on health-related quality of life and medical resource utilization of patients treated by allergy specialists and primary care providers. Ann Allergy Asthma Immunol 2002; 89: 139-147.

5 van Schayck $\mathrm{CP}$, van Weel C, Folgering $\mathrm{H}$, et al. Treatment of patients with airflow obstruction by general practitioners and chest physicians. Scand J Prim Health Care 1989; 7: 137-142.

Tada M, Kuraki T, Taooka Y, et al. Comparison of clinical management of young and elderly asthmatics by respiratory specialists and general practitioners. J Asthma 2015; 52: 162-169.

7 Bell D, Layton AJ, Gabbay J. Use of a guideline based questionnaire to audit hospital care of acute asthma. BMJ 1991; 302: 1440-1443.

Pearson MG, Ryland I, Harrison BD. Comparison of the process of care of acute severe asthma in adults admitted to hospital before and $1 \mathrm{yr}$ after the publication of national guidelines. Respir Med 1996; 90: 539-545. Pellicer C, Ramírez R, Perpiñá M, et al. anancia, pérdida y concordancia en el diagnóstico de asma entre neumólogos y no neumólogos [Gain, loss and agreement between respiratory specialists and generalists in the diagnosis of asthma]. Arch Bronconeumol 2001; 37: 171-176.

Pavord ID, Beasley R, Agusti A, et al. After asthma: redefining airways diseases. Lancet 2018; 391: 350-400.

Holguin F, Cardet JC, Chung KF, et al. Management of severe asthma: a European Respiratory Society/ American Thoracic Society guideline. Eur Respir J 2020; 55: 1900588.

Agache I, Akdis CA, Akdis M, et al. EAACI biologicals guidelines-recommendations for severe asthma. Allergy 2021; 76: 14-44. 
123 Morrow S, Daines L, Wiener-Ogilvie S, et al. Exploring the perspectives of clinical professionals and support staff on implementing supported self-management for asthma in UK general practice: an IMP²ART qualitative study. NPJ Prim Care Respir Med 2017; $27: 45$.

124 Kennedy A, Rogers A, Bower P. Support for self-care for patients with chronic disease. BMJ 2007; 335: 968-970.

125 Ivers N, Jamtvedt G, Flottorp S, et al. Audit and feedback: effects on professional practice and healthcare outcomes. Cochrane Database Syst Rev 2012; 6: CD000259.

126 Bravata DM, Sundaram V, Lewis R, et al. Closing the Quality Gap: A Critical Analysis of Quality Improvement Strategies (Vol. 5: Asthma Care). Rockville, Agency for Healthcare Research and Quality; 2007.

127 Pinnock H, Epiphaniou E, Pearce G, et al. Implementing supported self-management for asthma: a systematic review and suggested hierarchy of evidence of implementation studies. BMC Med 2015; $13: 127$.

128 Crespo-Gonzalez C, Fernandez-Llimos F, Rotta I, et al. Characterization of pharmacists' interventions in asthma management: a systematic review. J Am Pharm Assoc (2003) 2018; 58: 210-219.

129 Dexheimer JW, Borycki EM, Chiu KW, et al. A systematic review of the implementation and impact of asthma protocols. BMC Med Inform Decis Mak 2014; 14: 82.

130 McCleary N, Andrews A, Buelo A, et al. IMP2ART systematic review of education for healthcare professionals implementing supported self-management for asthma. NPJ Prim Care Respir Med 2018; $28: 42$.

131 Matui P, Wyatt JC, Pinnock H, et al. Computer decision support systems for asthma: a systematic review. NPJ Prim Care Respir Med 2014; 24: 14005.

132 Agustí A, Bafadhel M, Beasley R, et al. Precision medicine in airway diseases: moving to clinical practice. Eur Respir J 2017; 50: 1701655.

133 Baldacci S, Simoni M, Maio S, et al. Prescriptive adherence to GINA guidelines and asthma control: an Italian cross sectional study in general practice. Respir Med 2019; 146: 10-17.

134 Akinbami LJ, Salo PM, Cloutier MM, et al. Primary care clinician adherence with asthma guidelines: the National Asthma Survey of Physicians. J Asthma 2020; 57: 543-555.

135 Cloutier MM, Salo PM, Akinbami LJ, et al. Clinician agreement, self-efficacy, and adherence with the guidelines for the diagnosis and management of asthma. J Allergy Clin Immunol Pract 2018; 6: 886-894.

136 Eguiluz-Gracia I, van den Berge M, Boccabella C, et al. Real-life impact of COVID-19 pandemic lockdown on the management of pediatric and adult asthma: a survey by the EAACI Asthma Section. Allergy 2021; in press [https://doi.org/10.1111/all.14831]

137 Papadopoulos NG, Custovic A, Deschildre A, et al. Impact of COVID-19 on pediatric asthma: practice adjustments and disease burden. J Allergy Clin Immunol Pract 2020; 8: 2592-2599.

138 Pinnock H, Bawden R, Proctor S, et al. Accessibility, acceptability and effectiveness of telephone reviews for asthma in primary care: randomised controlled trial. BMJ 2003; 326: 477-479.

139 Pinnock H, Adlem L, Gaskin S, et al. Accessibility, clinical effectiveness and practice costs of providing a telephone option for routine asthma reviews: phase IV controlled implementation study. $\mathrm{Br} J$ Gen Pract 2007; 57: 714-722.

140 Butler SM. After COVID-19: thinking differently about running the health care system. JAMA 2020; 323 2450-2451.

141 Marlow J, O'Shaughnessy J, Keogh B, et al. Learning from a pandemic: how the post-covid NHS can reach its full potential. BMJ 2020; 371: m3867.

142 Schwamm LH, Estrada J, Erskine A, et al. Virtual care: new models of caring for our patients and workforce. Lancet Digit Health 2020; 2: e282-e285.

143 Shachar C, Engel J, Elwyn G. Implications for telehealth in a postpandemic future: regulatory and privacy issues. JAMA 2020; 323: 2375-2376.

144 Kropf M, Modre-Osprian R, Hayn D, et al. Telemonitoring in heart failure patients with clinical decision support to optimize medication doses based on guidelines. Annu Int Conf IEEE Eng Med Biol Soc 2014; 2014: 3168-3171.

145 Artanian V, Ross HJ, Rac VE, et al. Impact of remote titration combined with telemonitoring on the optimization of guideline-directed medical therapy for patients with heart failure: internal pilot of a randomized controlled trial. JMIR Cardio 2020; 4: e21962. 